\title{
Classification and Response Criteria in Mast Cell Disorders: Time to Revise?
}

\author{
Peter Valent $^{a}$ Hans-Peter Horny ${ }^{b}$ \\ ${ }^{a}$ Department of Internal Medicine I, Division of Hematology and Hemostaseology, Medical University of Vienna,

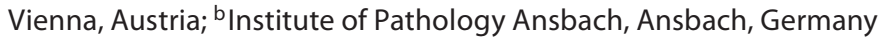

Dear Sir,

The purpose of our letter is to discuss and explore whether there is a need to refine the WHO classification of mastocytosis, disease-related criteria, and/or the response criteria that have been defined by the consensus group between 2000 and 2010.

Mastocytosis is a heterogenous group of disorders caused by an accumulation of mast cells in the skin and/or other organ systems. For many years, the exact relationship between mastocytosis and other hematopoietic disorders remained unclear and no robust diagnostic parameters were known. This changed by the recognition that mast cells are hematopoietic cells that form a separate lineage within the myelopoietic cell system $[1,2]$, and by identification of immunological, molecular, and biochemical markers of neoplastic mast cells [3-6]. In the late 1990s, European and US experts started a discussion on preexisting classification proposals and the possibility to update and merge these proposals into a consensus classification using novel molecular and biochemical markers and resulting criteria [7]. The resulting concept was discussed extensively in the Year 2000 Working Conference on Mastocytosis in Vienna (August 31 to September 3,2000 ), and was adopted by the WHO, leading to the official WHO classification of mast cell disorders $[8,9]$. Since then, the prognostic significance and value of this classification have been confirmed in diverse observational and clinical studies [10-12]. Later, in 2005, the EU-US Consensus Group also defined response criteria for indolent and aggressive disease variants [13] and refined the classification proposal [14]. In 2008, an updated version of the WHO classification of hematopoietic neoplasms was published [15]. Although this update did not adopt all recommendations proposed by the EU-US Consensus Group [14] the conceptual basis essentially remained unchanged [15]. So far, no alternative concept for the classification and for response evaluation in mastocytosis has been presented.

Nevertheless, we have the opinion that there is a need to refine both the classification and the response criteria in forthcoming years for a number of reasons. First, several new molecular, genetic, and immunological markers for the diagnosis of mastocytosis are available. Likewise, apart from KIT mutants, also other (KIT-independent) pathways and targets may play a critical role in disease progression in advanced mastocytosis [16]. In particular, KIT D816V is a mutant detectable in all categories of systemic mastocytosis (SM) including indolent SM (ISM) with normal life expectancy, but also aggressive SM (ASM) and mast cell leukemia (MCL), the leukemic variant of advanced SM defined by a poor prognosis and a short survival time [3, 4, 17-19]. Therefore, novel markers and target pathways sufficient to separate ISM from advanced SM (ASM/MCL) need to be defined. One potential pathway may lead to LYN activation and/or BTK activation in neoplastic mast cells in advanced disease [16]. However, in this pathway, the primary defect remains to be identified. Another marker is the CD30 antigen, which is preferentially expressed by mast cells in ASM and MCL, but is not expressed (or expressed weakly) by mast cells in patients with ISM [20]. Again, the molecular mechanisms and primary defects that contribute to CD30 expression in neoplastic mast cells in high-grade SM remain unknown. Once revealed, such novel molecular markers may serve as essential basis for refining/revising the existing classification of mastocytosis. Whatever will be a decisive new diagnostic marker, the preferential expression of CD30 in mast cells in ASM and MCL suggests that both conditions are closely related disease variants. This is also consistent with the notion that ASM and MCL are indistinguishable by histological and phenotypic criteria. Finally, ASM often presents with

\section{KARGER}

Fax +41613061234 E-Mail karger@karger.ch www.karger.com
Correspondence to: Prof. Dr. Peter Valent

Department of Internal Medicine I, Division of Hematology and Hemostaseology Ludwig Boltzmann Cluster Oncology, Medical University of Vienna

Währinger Gürtel 18-20, AT-1090 Vienna (Austria)

Tel. +43 140400 6085, Fax +43140400 4030,E-Mail peter.valent@ meduniwien.ac.at 
an increased number of mast cells in bone marrow smears and frequently progresses to MCL. In contrast to ASM, the percentage of mast cells in the bone marrow smear in MCL is $\geq 20 \%$, which is the only defined discriminative feature/criterion (ASM vs. MCL) $[8,9,14,15]$.

There are also other known molecular defects that have been identified in patients with SM, including the JAK2 V617F mutant, FIP1L1/PDGFRA, or AML1/ETO $[8,9,14,15]$. However, contrasting LYN, $\mathrm{BTK}$, or CD30 expression, these targets are indicative of an associated clonal hematological non-mast cell lineage disease (AHNMD). In these patients, a separate category of SM (SM-AHNMD) has been defined $[8,9,14]$, and there is no need to revise or refine the classification in this regard. Notably, the obvious advantage of this category is that the WHO frequently updates classifications and criteria, so that the AHNMD part in SM is automatically updated as well. However, as mentioned before, ASM and MCL may develop independently of an AHNMD and the molecular defects causing transformation into ASM or MCL remain to be identified.

There are also other unresolved issues that need to be addressed in the classification of SM in the future. For example, whereas the $\mathrm{WHO}$ has adopted several myelodysplastic/myeloproliferative overlap disorders, they did not adopt the myelomastocytic overlap (SM criteria not fulfilled despite overwhelming mast cell involvement in advanced myelodysplastic syndrome or acute myeloid leukemia) [8, $21,22]$. Moreover, a number of potential or provisional categories of SM, such as bone marrow mastocytosis, smouldering SM, splenic SM, occult SM, or well-differentiated SM, need to be discussed [14, 23]. The term 'occult mastocytosis' is used for a number of different conditions including mastocytosis obscured by an AHNMD and only discovered after cytoreductive therapy [24] and ISM presenting with small-sized mast cell infiltrates that are overlooked unless appropriate immunohistochemical and molecular tests are applied. A recently emerging issue is the relationship between SM and the so-called monoclonal mast cell activation syndrome, a condition defined by the presence of 1 or 2 minor SM criteria including KIT D816V and symptoms of mast cell activation $[25,26]$. Although there is a relationship to SM and some of the patients may indeed develop SM, it remains open whether monoclonal mast cell activation syndrome should be regarded as a subcategory of SM or as a separate mast cell disorder. Finally, the classification and criteria of cutaneous mastocytosis may need to be discussed and refined in the future.

An important reason for revising criteria could be improved therapeutic options. In particular, several new targeted drugs and treatment approaches are available, and the responses to certain drugs that can be achieved in clinical trials in the future may exceed responses seen with currently available drugs. However, so far, responses to cytotoxic and targeted drugs in ASM and MCL remain unsatisfactory. Likewise, no drug approach leads to complete hematological responses with complete disappearance of mast cell lesions and decrease of serum tryptase to normal in ASM or MCL. Therefore, response criteria still have to focus on cytoreduction and the improvement (or even normalization) of organ function $[13,14]$. An important aspect in this regard is that mastocytosis is a complex disease, and not all symptoms are caused by local destructive growth of mast cells. Therefore, response evaluation must not follow a simple multiparameter approach mixing symptoms of aggressive disease and mediator-related symptoms into one response category as has been proposed by others [27]. Likewise, mediator-related symptoms may improve although the patient progresses into MCL, or vice versa, mediator-related symptoms may worsen despite an improvement in organopathy caused by the mast cell infiltrate. Here, it is of importance to note that pathways and targets controlling the release of mediators in mast cells are not identical to that controlling mast cell growth and survival, even if some of the available drugs like PKC412 (midostaurin) may block both survival and mediator secretion in (normal and neoplastic) mast cells [28]. Thus, based on the complexity of the disease, it seems clear that symptoms and findings in mastocytosis and their responses to (targeted) drugs should always be evaluated separately and in a stepwise approach [14]. In a first step, the effect of the drug on organopathy caused by the local mast cell infiltration (so-called CFinding) is determined [14]. It is important to stress that stable disease has to be judged as success in patients with high-grade SM. In case of a response, the second question is whether the mast cell burden also decreased or whether the response was only a (pure) clinical response [14]. Mediatorrelated symptoms and osteoporosis/osteopenia need to be evaluated separately as these symptoms are usually not life-threatening and can be managed quite effectively using antimediator-type drugs [8-11, 14]. Notably, most of these patients have ISM and usually their disease will not require cytoreductive therapy [14]. Responses in these patients can still be heterogeneous and may be organ-specific. Likewise, in one and the same patient, anaphylaxis may improve during therapy whereas skin lesions may progress, or vice versa. One and the same patient may develop an ulcerative gastrointestinal disease during therapy although osteoporosis is clearly improving. There are further examples like this, and from all these observations it seems clear that response evaluation in SM is a complex issue and must follow established criteria, and it is important to measure and document responses to certain drugs (histamine receptor blockers, immunotherapy, bisphosphonates, others) separately for each organ system. Respective guidelines including proposals for grading of symptoms and response evaluation worked out by the consensus group are available and should be applied [14]. We recommend that such response evaluation should be performed in each case and that the documented improvement in mediator-related symptoms should be included as important endpoint in clinical trials.

In summary, we believe that the existing classification, diagnostic criteria, and response criteria prepared and defined by the consensus group between the years 2000 and 2010 are still valid and sufficient to classify and manage all patients with mastocytosis. Due to the complexity of the disease, however, it may sometimes be difficult, especially for the nonexperienced clinician, to apply criteria. Therefore, all patients with advanced mastocytosis should be referred to specialized centers. In the future, it may be appropriate to refine diagnostic and response criteria in a consensus approach and consensus proposal and to include novel molecular markers and parameters if possible.

\section{Acknowledgments}

This work was in part supported by a mastocytosis grant of the Medical University of Vienna. 


\section{References}

1 Valent P, Ashman LK, Hinterberger W, et al: Mast cell typing: demonstration of a distinct hematopoietic cell type and evidence for immunophenotypic relationship to mononuclear phagocytes. Blood 1989;73:1778-1785.

2 Valent P, Bettelheim P: Cell surface structures on human basophils and mast cells: biochemical and functional characterization. Adv Immunol 1992;52:333-423.

-3 Nagata H, Worobec AS, Oh CK, Chowdhury BA, Tannenbaum S, Suzuki Y, Metcalfe DD: Identification of a point mutation in the catalytic domain of the protooncogene c-kit in peripheral blood mononuclear cells of patients who have mastocytosis with an associated hematologic disorder. Proc Natl Acad Sci USA 1995;92:10560-10564.

4 Longley BJ, Tyrrell L, Lu SZ, et al: Somatic c-KIT activating mutation in urticaria pigmentosa and aggressive mastocytosis: establishment of clonality in a human mast cell neoplasm. Nat Genet 1996;12:312-314.

$\checkmark 5$ Schwartz LB, Sakai K, Bradford TR, Ren S, Zweiman B, Worobec AS, Metcalfe DD: The alpha form of human tryptase is the predominant type present in blood at baseline in normal subjects and is elevated in those with systemic mastocytosis. J Clin Invest 1995;96: 2702-2710.

-6 Escribano L, Orfao A, Díaz-Agustin B, et al: Indolent systemic mast cell disease in adults: immunophenotypic characterization of bone marrow mast cells and its diagnostic implications. Blood 1998;91:27312736.

7 Valent P, Escribano L, Parwaresch RM, et al: Recent advances in mastocytosis research. Summary of the Vienna Mastocytosis Meeting 1998. Int Arch Allergy Immunol 1999; 120:1-7.

8 Valent P, Horny HP, Escribano L, et al: Diagnostic criteria and classification of mastocytosis: a consensus proposal. Leuk Res 2001; 25:603-625.

9 Valent P, Horny H-P, Li CY, et al: Mastocytosis - mast cell disease; in Jaffe ES, Harris NL, Stein H, Vardiman JW (eds): World Health Organization (WHO) Classification of Tumours. Pathology and Genetics. Tumours of Haematopoietic and Lymphoid Tissues. Lyon, IARC Press, 2001, vol 1, pp 291-302.
0 Pardanani A, Lim KH, Lasho TL, et al: Prognostically relevant breakdown of 123 patients with systemic mastocytosis associated with other myeloid malignancies. Blood 2009;114:3769-3772.

11 Escribano L, Alvarez-Twose I, Sánchez-Muñoz L, et al: Prognosis in adult indolent systemic mastocytosis: a long-term study of the Spanish Network on Mastocytosis in a series of 145 patients. J Allergy Clin Immunol 2009; 124:514-521.

12 Lim KH, Tefferi A, Lasho TL, et al: Systemic mastocytosis in 342 consecutive adults: survival studies and prognostic factors. Blood 2009;113:5727-5736.

13 Valent P, Akin C, Sperr WR, et al: Aggressive systemic mastocytosis and related mast cell disorders: current treatment options and proposed response criteria. Leuk Res 2003. 27:635-641

14 Valent P, Akin C, Escribano L, et al: Standards and standardization in mastocytosis: consensus statements on diagnostics, treatment recommendations and response criteria. Eur J Clin Invest 2007;37:435-453.

15 Horny HP, Akin C, Metcalfe DD, et al: Mastocytosis (mast cell disease); in Swerdlow SH, Campo E, Harris NL, Jaffe ES, Pileri SA, Stein H, Thiele J, Vardiman JW (eds) World Health Organization (WHO) Classification of Tumours. Pathology and Genetics. Tumours of Haematopoietic and Lymphoid Tissues. Lyon, IARC Press, 2008, vol 2, pp 54-63.

16 Gleixner KV, Mayerhofer M, Rix U, et al: Delineation of a KIT-independent oncogenic pathway in neoplastic mast cells that involves Lyn and Btk and can be disrupted by the KIT/Lyn/Btk-targeting drug dasatinib. Blood 2007;110:460a-461a.

17 Fritsche-Polanz R, Jordan JH, Feix A, et al: Mutation analysis of C-KIT in patients with myelodysplastic syndromes without mastocytosis and cases of systemic mastocytosis. Br J Haematol 2001;113:357-364.

18 Féger F, Ribadeau Dumas A, Leriche L, Valent $\mathrm{P}$, Arock M: Kit and c-kit mutations in mastocytosis: a short overview with special reference to novel molecular and diagnostic concepts. Int Arch Allergy Immunol 2002; 127:110-114.
19 Garcia-Montero AC, Jara-Acevedo M, Teodosio C, et al: KIT mutation in mast cells and other bone marrow hematopoietic cell lineages in systemic mast cell disorders: a prospective study of the Spanish Network on Mastocytosis (REMA) in a series of 113 patients. Blood 2006;108:2366-2372.

20 Sotlar K, Cerny-Reiterer S, Petat-Dutter K, Hessel H, Müllauer L, Valent P, Horny HP: Aberrant expression of CD30 in neoplastic mast cells in high grade mastocytosis. Modern Pathol, in press.

21 Valent P, Sperr WR, Samorapoompichit P, et al: Myelomastocytic overlap syndromes: biology, criteria, and relationship to mastocytosis. Leuk Res 2001;25:595-602.

22 Sperr WR, Drach J, Hauswirth AW et al: Myelomastocytic leukemia: evidence for the origin of mast cells from the leukemic clone and eradication by allogeneic stem cell transplantation. Clin Cancer Res 2005;11:67876792

23 Valent P, Akin C, Sperr WR, Horny HP, Metcalfe DD: Smouldering mastocytosis: a novel subtype of systemic mastocytosis with slow progression. Int Arch Allergy Immunol 2002;127:137-139.

24 Bernd HW, Sotlar K, Lorenzen J, et al: Acute myeloid leukaemia with $\mathrm{t}(8 ; 21)$ associated with 'occult' mastocytosis. Report of an unusual case and review of the literature. J Clin Pathol 2004;57:324-328.

-25 Sonneck K, Florian S, Müllauer L, Wimazal F, Födinger M, Sperr WR, Valent P: Diagnostic and subdiagnostic accumulation of mast cells in the bone marrow of patients with anaphylaxis: monoclonal mast cell activation syndrome. Int Arch Allergy Immunol 2007;142:158-164

26 Akin C, Scott LM, Kocabas CN, KushnirSukhov N, Brittain E, Noel P, Metcalfe DD: Demonstration of an aberrant mast-cell population with clonal markers in a subset of patients with 'idiopathic' anaphylaxis. Blood 2007;110:2331-2333.

27 Pardanani A, Tefferi A: A critical reappraisal of treatment response criteria in systemic mastocytosis and a proposal for revisions. Eur J Haematol 2010;84:371-378.

28 Krauth MT, Mirkina I, Herrmann H, et al: Midostaurin (PKC412) inhibits immunoglobulin E-dependent activation and mediator release in human blood basophils and mast cells. Clin Exp Allergy 2009;39:17111720 . 